\title{
The perceptions, attitudes and knowledge of physiotherapy and chiropractic students regarding each others' professional practice
}

\begin{abstract}
Introduction: The roles of physiotherapists and chiropractors demonstrate some overlap. Both are members of a multidisciplinary team and contribute to the holistic care of patients. Good understanding of each others' professional practice may lead to good working relationships with effective referrals, interdisciplinary and multidisciplinary management of patients.

Purpose: To investigate the perceptions, attitudes and knowledge of undergraduate physiotherapy and chiropractic students about each others' professional roles in clinical practice.
\end{abstract}

Methodology: Data was obtained using a questionnaire with closed and open-ended questions. First and final-year physiotherapy $(n=72)$ and chiropractic students $(n=49)$ participated.

Results: First and final year physiotherapy students scored $58 \%$ and $62 \%$ respectively in the "test" on chiropractic; chiropractic students scored $52 \%$ and $68 \%$ respectively in the test on physiotherapy. Seventy percent of the chiropractic and $14 \%$ of the physiotherapy students had visited the practice of other profession. Sixty seven percent of the chiropractic and $38 \%$ of the physiotherapy students found it was effective. Forty seven percent chiropractic and $80 \%$ physiotherapy students considered physiotherapy and chiropractic to be in direct competition. Sixty six percent of the chiropractic students and 49\% of the physiotherapy students expressed the intention of working together with the other profession.

Discussion: The reason for the possible feelings of competitiveness could be because in South Africa there is vast overlap of practice in both professions; Physiotherapists and chiropractors are seen to use modalities that are similar. This may be viewed as an indication of the importance in defining the roles, scope and characteristics of both physiotherapy and chiropractic.

Conclusion: The knowledge of the physiotherapy and chiropractic students is equal, however, chiropractic students have more positive perceptions and attitudes towards physiotherapy than physiotherapy students have of chiropractic.

KEYWORDS: PHYSIOTHERAPY, CHIROPRACTIC, HEALTH KNOWLEDGE, ATTITUDES, PROFESSIONAL PRACTICE.

\section{INTRODUCTION}

A characteristic of any profession is that it occupies a social standing relative

\section{Correspondence to: \\ Nirmala Naidoo \\ Discipline of Physiotherapy \\ School of Physiotherapy, \\ Sports Science and Optometry \\ Faculty of Health Science \\ University of KwaZulu-Natal \\ Private Bag X54001 \\ Durban 4000 \\ South Africa \\ Tel: +27312607817 \\ Fax: +2731 2608106 \\ Email: naidooni@ukzn.ac.za}

to other professions (Turner, 2001). The professional relationship between physiotherapists and chiropractors is important as both physiotherapists and chiropractors are members of a multidisciplinary team and contribute to the holistic care of patients. Understanding the perceptions and attitudes of undergraduate students, (who are a key target because they are future practitioners), would help the profession create more effective marketing strategies, knowing the misconceptions that need to be corrected (Prati and Liu, 2006). This study was undertaken to determine the perceptions, attitudes and knowledge that Physiotherapy and chiropractic students have of each others' professional practice.
1.1 Physiotherapy Education and Practice Physiotherapy is concerned with 'assessing, treating and preventing movement disorders, restoring normal function or minimising dysfunction and pain in adults and children with physical impairment, to enable them to achieve the highest possible level of independence; preventing recurring injuries and disability in the workplace, at home, or during recreational activities and promoting community health for all age groups (South African Society of Physiotherapy, 2008). Physiotherapists use 'skilled evaluation, skilled hands on therapy such as mobilisation, manipulation, massage and acupressure; individually designed exercise 
programmes, relaxation techniques, sophisticated equipment, hydrotherapy and biofeedback, specialised electrotherapy equipment, heat, ice and traction to relieve pain and assist healing and recovery, suitable walking aids, splints and appliances, patient education' (South African Society of Physiotherapy, 2008).

Physiotherapy as a profession began during World War I. In an effort to provide early rehabilitation to wounded soldiers, the Surgeon General's office formed the Division of Special Hospitals and Physical Reconstruction in France. This division created "Reconstruction Aides", (later known as Physiotherapists). Physiotherapy then began to grow as a recognised medical profession until the poliomyelitis epidemic in the 1940's and 50 's. The role of physiotherapy became increasingly important as physiotherapists became essential in helping people with polio to minimise or overcome its paralysing effects. In 1921 a small group of masseurs in Cape Town formed the Certified Masseurs Association. In the same year a similar group in Natal formed a branch of the United Kingdom Chartered Society of Massage and Remedial Gymnastics. These two groups then formed the South African Society of Massage and Medical Gymnastics in December 1924. In 1929 the South African Society published its first journal, and in 1932 they changed their name to the South African Society of Physiotherapists. After the Second World War they changed it again to the South African Society of Physiotherapy (SASP). The original Physiotherapy programme was a 3-year diploma; this later changed to a four-year degree programme. In South Africa there are eight Physiotherapy educational institutions. The Physiotherapy programme is conducted over four years and includes scientific, medical and clinical modules (Health Professions Council of South Africa, 2008).

\subsection{Chiropractic Education and Practice}

The Chiropractic Association of South Africa (2008) defines chiropractic as:

'A health profession specialising in the diagnosis, treatment and prevention of mechanical disorders of the musculoskeletal system and the affects of these disorders on the function of the nervous system and general health. Chiropractic practitioners essentially rely upon noninvasive treatment methods and will refer patients to medical practitioners should medication or surgery be indicated. This approach is further reinforced by chiropractors in their promotion of healthy lifestyles such as the avoidance of smoking and excess stress, proper diet and exercise.'

In South Africa, the first association was formed in 1939, namely the South African Manipulative Practitioners Association (SAMPA). This later became the Pan-African Chiropractors' Association (PACA). In 1952 the South African Chiropractors' Association (SACA) was formed, and later fused with PACA to form the Chiropractor's Association of South Africa, (CASA). In 1971 a government Bill was promulgated that closed the CASA register to chiropractors and chiropractic students. In 1982 the chiropractors, homeopaths and Allied Health Services Professions Council were formed, permitting legal chiropractic practice. The Chiropractic Registry was reopened in 1985 and the first students were accepted at Technikon Natal (presently Durban University of Technology) in 1989 (CASA, 2008). In South Africa, chiropractic can be studied at the Durban University of Technology, Durban, KwaZulu Natal and University of Johannesburg, Gauteng Province. Both programmes extend over six years and consist of two years of basic sciences followed by four years of specific chiropractic courses (Chiropractic Association of South Africa, 2008).

\subsection{Aim of the study}

The aim of this study was to investigate the perceptions, attitudes and knowledge of undergraduate physiotherapy and chiropractic students about professional practice. This would determine the understandings students have of the respective professions. Good understanding of each others' professions and roles could lead to good working relationships. This would lead to efficient referrals, interdisciplinary and multidisciplinary management of patients, whilst striving for quality and holistic patient care.

\section{Background}

A literature search of the databases PEDRO, Pubmed, SABINET and Cinahl revealed that no previous study to determine the perceptions, attitudes and knowledge of undergraduate physiotherapy and chiropractic students pertaining to professional practice was conducted.

A study on inter-professional perceptions of undergraduate health care students in the United Kingdom from medicine, nursing, dietetics, pharmacy and physiotherapy was conducted by Hind et al (2003). All rated their own profession as being caring with good communication with their patients. The pharmacists rated their profession higher than all the other professions rated their own.

Dalley and Sim (2001) investigated nurses' perceptions of physiotherapists as rehabilitation team members, using semi-structured interviews. The study revealed that nurses perceived the physiotherapists' role as 'being concerned with mobility and movement,' for which the physiotherapists' knowledge and skills were valued. They felt that physiotherapists did not really understand the demands and pressures of nursing and did not recognise their role as decisionmakers in rehabilitation. The nurses viewed each profession to play different roles in rehabilitation, despite some overlap. They perceived physiotherapy as 'specific and measurable', and nursing as 'generalised and undefined'. The nurses valued the physiotherapists' aptitude in mobility and their 'contribution to rehabilitation'. The researchers concluded that there are first "barriers of organisation," where the structured day of the physiotherapist showed an organisation the nurses did not feel they had; and also "barriers of relationships," where the nurses felt the physiotherapists had insufficient understanding of the stresses the nurses experience, which might be a "barrier to teamwork". A third category "Barriers relating to patients' perceptions" showed that patients perceive each profession as being different. Finally, "barriers to continuity of rehabilitation" revealed that rehabilitation was disrupted, possibly due to lack of instruction, therapists not 
working after hours or by lack of nursing time for rehabilitation.

The communication between general practitioners and chiropractors in the Netherlands was assessed by Brussee et al (2001). They reported that $78 \%$ of the general practitioners had heard of chiropractic, mainly from patients who had consulted a chiropractor (78\%) and that $10 \%$ refer patients to a chiropractor regularly. Referrals were found to be related to the general practitioners' knowledge of chiropractic and a statistically significant relationship was found between a positive opinion of previous communications and the frequency of patient referrals to chiropractors. More than $80 \%$ were interested in getting feedback reports from the chiropractor. The researchers report that although many general practitioners seem to have positive attitudes toward communication with chiropractors, there are barriers and deficiencies in current communications between them. Negative experiences, the use of confusing terminology, stereotyping and lack of knowledge about each others' professional practice seem to be the major factors influencing the communication process. The researchers conclude that most general practitioners have a 'neutral to positive attitude toward communication with chiropractors.'

A study to assess the perceptions of students in the health professions about inter-professional cooperation was conducted by Hawk et al (2002). Questionnaires were completed by students of medicine, nursing, social work, osteopathy, physical therapy, physician assistant, podiatry, and chiropractic. The investigators used the Interdisciplinary Education Perception Scale (IEPS) and found that physician assistant students had the most positive attitude toward all four factors assessing inter-professional association, and chiropractic students had the least. The IEPS is an 18-item questionnaire that uses a six-point Likert-type scale to measure attitudes toward interprofessional cooperation on four factors: competence and autonomy, perceived need for cooperation, perception of actual cooperation and understanding others' value (Hawk et al (2002).

Skjorshammer (2001) determined the inter-professional differences in perception and management of conflicts in a Norwegian hospital. Health professionals were interviewed and it was found that during conflict, there are three major approaches: avoidance, forcing and negotiation. Avoidance behaviour or suppression was the most common reaction, with the nurses and physicians differing significantly in their perception of conflict and when to react to it.

Inter-professional relationships between the different health care groups are not always ideal. Current perceptions and levels of awareness may be influenced by competition for patients and conflicts of opinion. If these different groups could develop a better working relationship this could ultimately lead to effective health care. Knowledge, perceptions and attitudes held by physiotherapy students of chiropractic students and that of chiropractic students of physiotherapy students could assist in developing strategies to correct possible misconceptions and stereotypes to enhance patient care.

\section{METHODS AND PROCEDURES}

The participants were all first year and final year physiotherapy $(n=72)$ and chiropractic students $(n=55)$ in Durban, KwaZulu-Natal. The physiotherapy students were from the University of KwaZulu-Natal (UKZN), Westville campus, Durban. The Chiropractic students were from the Durban University of Technology (DUT), Steve Biko campus, Durban. The total study population was 127 participants. As the physiotherapy undergraduate programme is four years and the chiropractic six years, the first and final year students from each programme participated in this study.

Questionnaires with both closed and open-ended questions were constructed. The first section of the questionnaire was constructed using information taken from the prospectus of each institution for 2006. The scope of each profession was the basis for a list of competencies. Additional items were added which are not in the scope of each profession were used as a test section to determine the knowledge of students about the others' profession. The second section of the questionnaire was developed with the view of determining the attitudes and perceptions of the students towards the other profession. The questionnaire was piloted for content validity using second year physiotherapy students. There were no changes made after the pilot study.

Ethical approval was obtained from the Ethical Committee at the University of KwaZulu-Natal. Permission was obtained from the Departments of Physiotherapy (UKZN) and Chiropractic (DUT) to conduct the study. Informed and signed consent was obtained from the participants prior to the administration of the questionnaires.

The data obtained from the questionnaires was collated. The quantitative data was subjected to statistical analysis with Microsoft Excel version 2007 using descriptive analyses. The qualitative data was categorised for positive and negative responses and grouped together with identified themes respectively.

\section{RESULTS}

Questionnaires were distributed to 72 physiotherapy students and 55 chiropractic students. Of the total sample of 127 questionnaires, 38 were completed by first year physiotherapy students, 34 by final year physiotherapy students, 29 by first year chiropractic students and 20 by final year chiropractic students. The return of questionnaires was $100 \%$ from physiotherapy students and 89\% for chiropractic students.

In the first year physiotherapy class, $8 \%$ were male and $92 \%$ female with the age range of 17 to 22 years and the average age 19 years. In the final year physiotherapy class, $15 \%$ were male and $85 \%$ female. The age range was 21 to 29 years and the average age 23 years. The first year chiropractic class had $30 \%$ males and $70 \%$ female students where the age range was 17 to 29 and the average age 23 years. Twenty five percent male and $75 \%$ female students were representative of the final year chiropractic class, with the age range of 22 to 31 and the average age of 24 years.

In the first section of the questionnaire, the students were tested on their knowledge of the other profession. Figure 1 illustrates the results obtained.

The responses to the open-ended questions regarding students' attitudes 
and perceptions about both professions being competitive with each other and whether they wished to work closely together are presented below.

\subsection{Overlap of skills and competitive- ness of both professionals}

Physiotherapy students agreed that there is competitiveness between both professions. The main reason cited for this is that "Physiotherapists and chiropractors do things that are similar" (49\% and $38 \%$ respectively first and final year physiotherapy students) and that "Chiropractors do everything originally done by physiotherapists (20\% and 26\% respectively). Four percent of the first year students felt "rehabilitation is not done by chiropractors." Final year physiotherapy students felt that the skills physiotherapists have are "more and different" with "treatment of the chest and musculoskeletal system." Six percent felt that "Chiropractors are doctors, but do things that are actually physiotherapy techniques."

Chiropractic students reported the following: $42 \%$ first year and $40 \%$ final year students felt that "physiotherapists treat in a different way" but "both treat similar things." Ten percent first years and 30\% final year students felt that both "can work together to complement each other." Seven percent of the first year students felt that "physiotherapists take our patients and treat musculoskeletal conditions." They also commented that physiotherapists are "no contest to chiropractic." The competitiveness is further borne out by "chiropractors can diagnose and physiotherapists cannot," the public is more aware of physiotherapists and doctors refer more to them." There is also the perception $15 \%$ final year students that physiotherapists are unwilling to work with chiropractors and that there is animosity between the professions.

\subsection{Students' overview of collaboration of both professions}

Forty five percent first year and 53\% final year physiotherapy students reported that they would like to work more closely with chiropractors. Fifty two percent first year and $80 \%$ final year chiropractic students agreed with them.
Some of the reasons stated by physiotherapy students for this decision are that "the two professions should be different," we could learn from each other and that "more patients would be referred to physiotherapy with the holistic care benefits if we work closely with chiropractors." Those who disagreed felt that they "do not believe in what chiropractors do" as "chiropractors infringe on our roles" and "do not approve of their treatment techniques as many are common to Physiotherapy."

First year Chiropractic students agreed that physiotherapists and chiropractors are "different and function differently" and that "patients could benefit from both professionals." Those

Figure 1: Graph representing the average percentage scored by students for their knowledge of the others' profession.

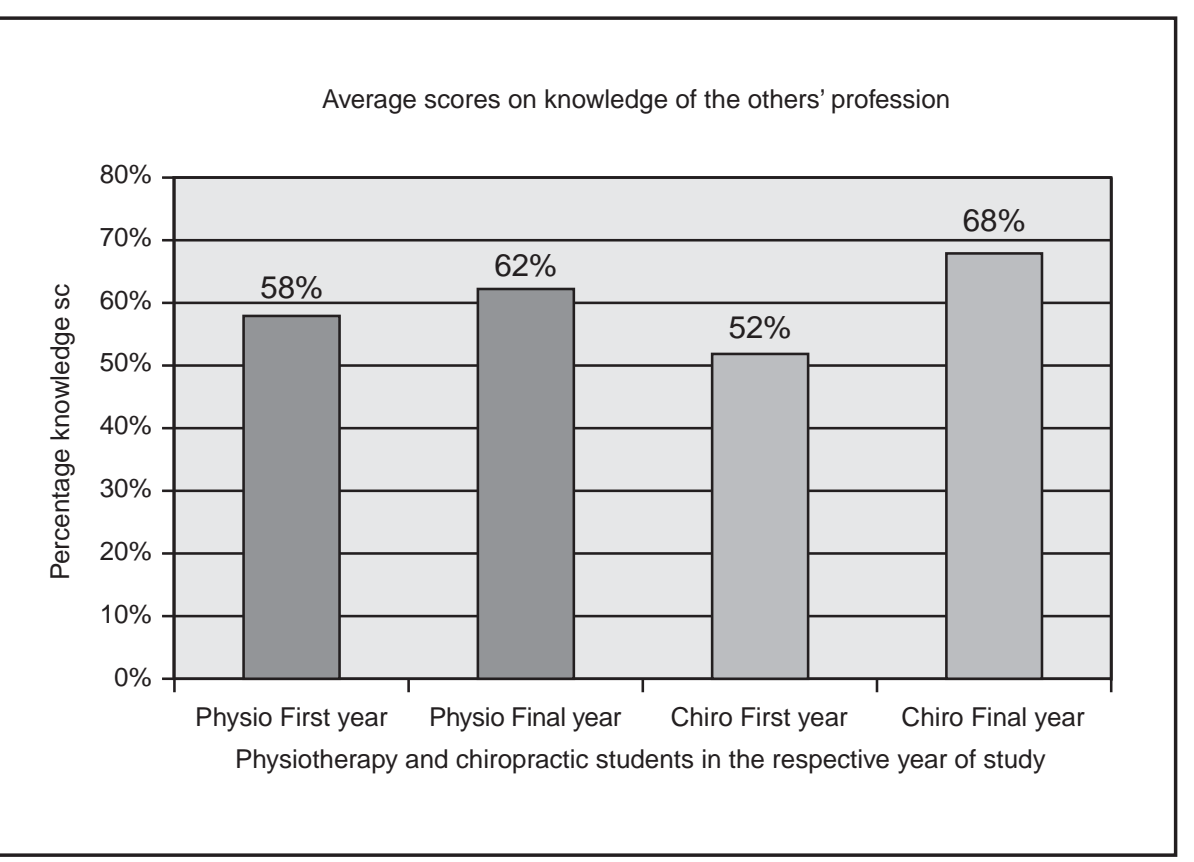

Figure 2: Graph showing the percentage of each class who perceived physiotherapy and chiropractic to be in direct competition.

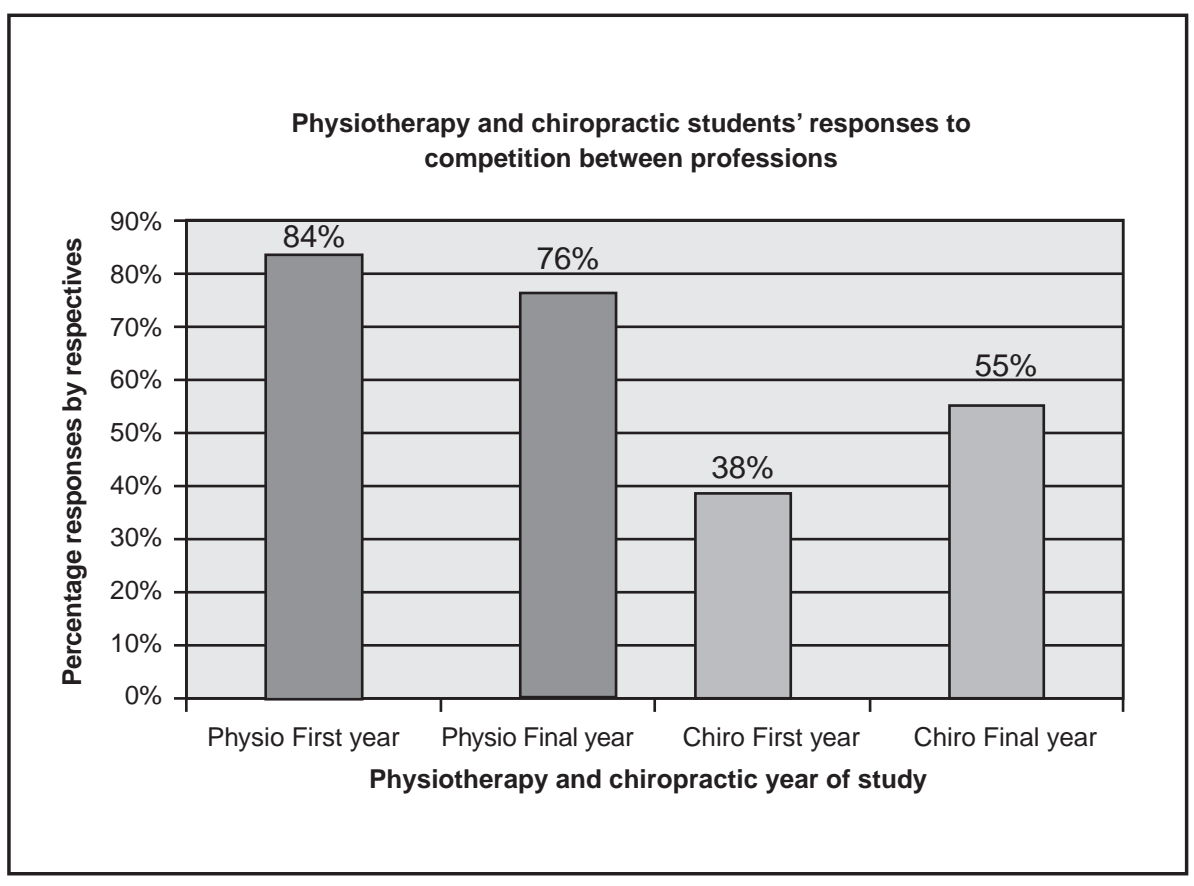


who disagreed with this reported that "chiropractors function independently; no need for Physiotherapy" and that physiotherapists and chiropractors do not believe in the same things." The final year students agreed that "both could learn from the physiotherapists." Some final year students disagreed and reported that "there is no need to work closely with physiotherapy" as there is "too much competition."

Forty five percent of the physiotherapy students reported that they would refer patients to the chiropractor. In contrast, $70 \%$ of the chiropractic students reported that they would refer patients for physiotherapy.

Some comments the chiropractic students made about physiotherapy and chiropractic where there could be improved working relations and collaboration include the following:

"Chiropractors and physiotherapists should work to complement and not to compete with each other"

"Chiropractic gives better relief of symptoms because they are excellent healers and it is a great profession"

"Chiropractors diagnose and treat whereas physiotherapists have to be referred to by a doctor" will complement each other" and "we

"Both professions are good courses" and the best option is "a combination of chiropractic and biokinetics."

Physiotherapy students listed some of the following suggestions for improved working relations with chiropractors as follows:

"Chiropractors take over the Physiotherapists job"

"Unfair that Chiropractors are called doctors; what about physiotherapists?"

"Both professions are equally important, but their domains should be separated"

"Would like to know more about chiropractors"

"Physiotherapy needs to be marketed more"

"There is place for both professions, therefore there is no need for competition"

"Both professions need more knowledge and information about each other."

\section{DISCUSSION}

There is rapid evolution of the Physiotherapy profession as well as that of the chiropractors. This has entrenched the understanding and need for a multi-

Table 1: Summary of physiotherapy and chiropractic students' perceptions and attitudes towards the others' profession.

\begin{tabular}{|c|c|c|c|c|}
\hline \multirow[t]{2}{*}{ Question } & \multicolumn{2}{|c|}{$\begin{array}{l}\text { Physiotherapy } \\
\text { students (\%) }\end{array}$} & \multicolumn{2}{|c|}{$\begin{array}{l}\text { Chiropractic } \\
\text { Students (\%) }\end{array}$} \\
\hline & $\begin{array}{l}1 \text { st } \\
\text { YEAR }\end{array}$ & $\begin{array}{l}\text { FINAL } \\
\text { YEAR }\end{array}$ & $\begin{array}{l}1 \text { st } \\
\text { YEAR }\end{array}$ & $\begin{array}{l}\text { FINAL } \\
\text { YEAR }\end{array}$ \\
\hline $\begin{array}{l}\text { Have you or a family } \\
\text { member ever consulted } \\
\text { with the other profession? }\end{array}$ & 16 & 12 & 79 & 70 \\
\hline $\begin{array}{l}\text { Was the treatment } \\
\text { effective? }\end{array}$ & 50 & 25 & 70 & 64 \\
\hline $\begin{array}{l}\text { Do you think physio- } \\
\text { therapy and chiropractic } \\
\text { are in direct competition? }\end{array}$ & 84 & 76 & 38 & 55 \\
\hline $\begin{array}{l}\text { Would you like to work } \\
\text { closely with the other } \\
\text { profession? }\end{array}$ & 45 & 53 & 52 & 80 \\
\hline $\begin{array}{l}\text { Would you refer a patient } \\
\text { to the other profession? }\end{array}$ & 63 & 26 & 55 & 85 \\
\hline
\end{tabular}

disciplinary approach to patient care. Consequently, Physiotherapists and chiropractors should be part of the team and therefore need to work closely. Few studies have evaluated the Physiotherapy profession relative to other occupations. This is a serious omission in terms of marketing, because the strength of the competition must be known and understood (Turner, 2001).

The increased focus on the multidisciplinary team approach to patient care may bring to light the necessity for multidisciplinary team teaching. A study to investigate undergraduate physiotherapy and podiatry students' stereotypes of each profession before and after a semester of inter-professional education was conducted by Mandy et al (2004), where they measured the stereotypes using the Health Team Stereotype Scale. The researchers found that stereotypical perceptions exist within the two professional groups and these could be improved after exposure to inter-professional education.

Kenny and Adamson (1992) conducted a study to determine the issues of dominance, autonomy and authority between medical doctors and other health professionals in Sydney, Australia. Interviews were conducted with a sample of nurses, physiotherapists, occupational therapists, speech pathologists and psychologists, inquiring of their perceptions on the interaction of the medical profession and their occupational group. The health professionals did not feel regarded by doctors as equals and the same percentage felt that doctors did not have sufficient knowledge and understanding of their professions. A majority felt that they have adequate autonomy and could discuss the instructions given by the doctor and also offer suggestions to doctors. The majority of physiotherapists reported that they had refused a doctor's instructions or recommendations at least six times in the past year, compared to $30 \%$ of occupational therapists, $35 \%$ of psychologists, $17 \%$ of nurses and $11 \%$ of speech pathologists. The researchers concluded that the results of their study "confirm that interprofessional conflict and competition continues to be feature in the health care system." The results of this study 
corroborate this finding of conflict amongst some healthcare professionals and the possible ways to address this would need to be investigated in order that patient care may not be compromised.

Both physiotherapy and chiropractic students had similar understandings of the others' profession. The final year students had better knowledge of the other profession than the first year students. This is contrary to the findings of Langworthy and Smink (2000) where most of the practitioners had limited knowledge of chiropractic. The reason that final year students have more knowledge of the others' profession compared to first year students in both professions may be because first year students may have had less exposure to the scope of both professions and may therefore be limited in their knowledge.

Fewer physiotherapy students had themselves or a family member seen a chiropractor and more chiropractic students and/or family member had sought the services of a physiotherapist. This would explain why the chiropractic students knew more about physiotherapy than physiotherapy students knew about chiropractic. The increased accessibility to physiotherapists may also play a part as there are practicing physiotherapists in both public hospitals and private care, whereas chiropractors are solely in private practice. Although this may be the case, some physiotherapy students felt that physiotherapy is not marketed adequately leaving the perception that chiropractors are ahead in this area.

Contrary to this study Langworthy and Smink (2000) reported $67 \%$ manual therapists and $19 \%$ of the physiotherapists perceived chiropractors as direct competition, while $45 \%$ osteopaths and $48 \%$ physiotherapists found chiropractic complementary to their professions, showing positive perceptions towards chiropractors by the physiotherapists. All osteopaths and 50\% manual therapists and physiotherapists reported that chiropractors were skilled practitioners. This may imply that these are practitioners work together closely. The authors found that greater awareness levels seem to correlate with increased levels of acceptance and respect. 'Current levels of communication and cooperation were thought to be poor to nonexistent although the majority welcomed closer links, particularly in relation to the treatment of spinal complaints'. The researchers conclude that "professions may wish to focus on perceived areas for potential co-operation." Practitioners may benefit from the educational process that would inevitably accompany these. In the long-term such an approach should be of benefit to both professions and patients in our care.

The first year chiropractic class recorded that physiotherapists treat in a different way to chiropractors, so they are not competition. This would explain the majority of chiropractic students reporting that physiotherapists and chiropractors should work together to complement each other. This shows a more positive attitude, implying that chiropractic students see physiotherapy more positively. Final year chiropractic class reported that both professions are excellent and should work together; both treat similar type of patients and that the public is more aware of physiotherapists and doctors refer more to them. These results also refute the findings of Hawk et al (2002) they reported that chiropractic students have the least positive attitude towards inter-professional association with other health professionals.

The majority of the final year chiropractic students said that working closer would benefit the patient more and a minority of the final year physiotherapy students said they do not agree with what chiropractors do. These attitudes may hinder communication and may impact on patient care. The majority of the chiropractic class (both first and final years) said they would refer a patient to a physiotherapist. A minority of the final year and majority of the first year Physiotherapy students would refer a patient to a chiropractor. This again indicates that the perceptions the chiropractic students have of physiotherapy is more positive. These attitudes and perceptions could imply the need for education and increased communication on the roles and scope of each profession to foster effective relationships for efficient multidisciplinary care of patients.

\section{CONCLUSION}

Physiotherapy and chiropractic students are generally aware of the roles of each others' professions. There was a greater understanding by chiropractic students as the majority had received or experienced Physiotherapy with good effect. The majority of Physiotherapy students felt that chiropractors and Physiotherapists are in direct competition; and the majority of chiropractic students would like to work more closely with Physiotherapists and are more likely to refer patients to Physiotherapy.

The differences in the perception and attitudes of both Physiotherapy and chiropractic students in this study reveals a need for more education and communication on the scope of both Physiotherapy and chiropractic. Physiotherapy and chiropractic students in this cohort reflect equal knowledge on the others profession, the chiropractic students have more positive perceptions and attitudes towards physiotherapy than physiotherapy students have of chiropractic.

\section{RECOMMENDATIONS}

It would be of interest to expand this study to physiotherapy and chiropractic students outside KwaZulu Natal to a national study.

Similar studies are advocated for qualified physiotherapists and chiropractors in KwaZulu-Natal and South Africa and to compare the results to those obtained from students.

Inter-professional education between physiotherapy and chiropractic students in KwaZulu -Natal may be valuable to foster interdisciplinary understanding and acceptance.

\section{LIMITATIONS}

The research cohort was confined to Physiotherapy and chiropractic students in KwaZulu-Natal. A national study would determine the influence and alignment of the national and international curricula in both professions. This would also assist with the clarification of the scope of practice and make explicit the roles of the different professions in order to impress awareness among the multidisciplinary team and the public to improve holistic patient care. 